\title{
Produtividade e características agronômicas da cana-de-açúcar em diferentes sistemas de preparo do solo
}

\author{
Emmerson Rodrigues de Moraes ${ }^{1}$, Luis Augusto da Silva Domingues², Matheus Henrinque \\ Medeiros³, Joicy Vitória Miranda Peixoto ${ }^{4}$, Regina Maria Quintão Lana ${ }^{3}$
}

\author{
${ }^{1}$ Instituto Federal de Educação, Ciência e Tecnologia Goiano - IFGoiano, Campus Morrinhos, Goiás, Brasil. E-mail: \\ emmerson.moraes@ifgoiano.edu.br \\ ${ }^{2}$ Instituto Federal de Educação, Ciência e Tecnologia do Triângulo Mineiro - IFTM, Uberlândia, Minas Gerais, Brasil. E-mail: \\ luisaugusto@iftm.edu.br \\ ${ }^{3}$ Universidade Federal de Uberlândia - UFU, Uberlândia, Minas Gerais, Brasil. E-mail: matheushm97@ outlook.com, \\ rmqlana@terra.com.br \\ ${ }^{4}$ Universidade Federal de Goiás - UFG, Goiânia, Goiás, Brasil. E-mail: joicyvmpeixoto@ yahoo.com.br
}

Recebido: 03/03/2016; Aceito: 24/03/2016.

\section{RESUMO}

O preparo do solo pode contribuir para a melhoria das condições físicas e químicas objetivando o adequado desenvolvimento da cultura da cana-de-açúcar. O objetivo foi avaliar a interferência de diversos tipos de preparo de solo e do plantio direto nas características agronômicas e produtividade da cana-de-açúcar em uma área de reforma. O experimento foi conduzido no município de Goianésia (GO) em um Latossolo Vermelho-Amarelo de textura média, utilizando-se a variedade IAC 87-3396. O delineamento experimental utilizado foi o de blocos casualizados com seis tratamentos e quatro repetições. Os tratamentos foram: T1) dessecação + calcário + aração + gradagem; T2) calcário + subsolador + gradagem; T3) dessecação + calcário + plantio direto; T4) dessecação + calcário + subsolador; T5) destruidor de soqueira + calcário + subsolagem; e, T6) destruidor de soqueira + calcário + grade + aração + grade. Foram avaliados o número, diâmetro, altura e a produtividade dos colmos. Os diferentes preparos de solo não influenciaram nas características agronômicas da cana-planta. $\mathrm{O}$ uso de arado de aiveca associado à grade na incorporação do calcário proporciona as maiores produtividades de colmo de cana-de-açúcar. O plantio direto mostrou-se alternativa viável ao cultivo da cana-de-açúcar, por apresentar produtividade de colmo semelhante aos demais preparos do solo.

Palavras-chave: plantio convencional, plantio direto, revolvimento do solo, Saccharum spp.

\section{Productivity and agronomic characteristics of sugarcane under different soil tillage systems}

\begin{abstract}
The soil preparation may contribute to improvement of the physical and chemical conditions aiming for proper development of the sugarcane crop. The objective was to evaluate the effect of types of soil preparation and tillage on agronomic characteristics and productivity of sugarcane in a reform area. The experiment was conducted in Goianésia (GO) in a Yellow-Red Latosol of medium texture, using the variety IAC 87-3396. The experimental design was randomized blocks with six treatments and four replications. The treatments were: T1) desiccation + lime + plowing + disking; T2) lime + subsoiling + disking; T3) desiccation + lime + tillage; T4) desiccation + lime + subsoiling; T5) ratoon destroyer + lime + subsoiling; and, T6) ratoon destroyer + lime + grade + plowing + grade (T6). The number, diameter, height and productivity of sugarcane were measured. Different soil tillage did not affect the agronomic characteristics of the plant cane. The use of moldboard plow associated with the grid in lime incorporation provides the highest yields of sugarcane. No-tillage system shown to be a viable alternative to the cultivation of sugarcane, to present stem productivity similar to other soil preparation.
\end{abstract}

Key words: conventional tillage, no-tillage, soil tillage, Saccharum spp. 


\section{Introdução}

A cana-de-açúcar (Saccharum spp.) é uma das principais culturas agrícolas da economia brasileira. $\mathrm{O}$ Brasil é o maior produtor mundial de cana-de-açúcar, sendo responsável por mais da metade de todo o açúcar comercializado no mundo, além de ser o maior exportador de etanol. O estado de São Paulo é o maior produtor nacional de cana-de-açúcar, detendo $52 \%$ da área plantada, seguido de Goiás, Minas Gerais e Mato Grosso do Sul. A estimativa de colheita para a safra 2026/17 pode chegar a 658,7 milhões de toneladas, cerca de $3,8 \%$ maior que a produção da safra anterior (CONAB, 2015).

Nos últimos anos, a cultura da cana-de-açúcar tem apresentado significativos aumentos de produtividade na região Centro-Sul do Brasil. Este aumento é resultado de melhoramento genético, conhecimento e uso de técnicas agronômicas que melhor se adaptam à cultura e que permitem uma melhor expressão de seu potencial genético (CHAVES et al., 2015).

O cultivo de cana-de-açúcar está se expandindo para áreas, anteriormente, ocupadas com pastagem com algum nível de degradação. De acordo com Inácio e Santos (2013), o cultivo da cana-de-açúcar está se sobrepondo a outras atividades agrícolas, como a pecuária, cafeicultura e sojicultura. Estes autores caracterizam áreas de expansão aquelas que nas safras anteriores eram destinadas a outros cultivos e na safra corrente cultivada com cana-de-açúcar estando disponíveis para primeira colheita. Lavouras de canade-açúcar que foram convertidas em outro uso por um período igual ou maior a duas safras e voltaram a ser cultivadas com cana-de-açúcar também se inserem nessa classe.

Segundo Neves e Conejero (2007), o sistema produtivo de cana-de-açúcar é complexo, pois necessita de fornecedores de matéria-prima e consumidores produtores de biocombustíveis, fertilizantes, rações, e produção de alimentos. Sendo assim, o planejamento das atividades envolvidas com a cultura da cana-deaçúcar, desde o plantio até sua colheita, é uma etapa extremamente importante na sua exploração econômica. Atualmente, estudos e avaliações de sistemas de cultivo de cana-de-açúcar devem objetivar a análise de todos os componentes de produção, inclusive aqueles envolvidos com os custos de implantação.

São inúmeros os questionamentos sobre qual preparo de solo deve ser adotado visando à melhoria da produtividade e sustentabilidade do canavial. Os preparos convencionais e o manejo conservacionista tradicionalmente chamado de "plantio direto" necessitam de adaptações pois, sendo esta cultura semiperene, a rotação de culturas prevista anualmente no plantio direto não ocorre. Quando há rotação com espécies leguminosas após vários cortes do canavial, para iniciar-se novo plantio da cana-de-açúcar há a necessidade de abertura de grandes sulcos visando colocar os colmos (toletes) caracterizando-se ainda mais o chamado cultivo mínimo e descaracterizando o plantio direto (ARRUDA et al., 2015).

O preparo do solo promove o seu revolvimento expondo-o às ações do ambiente como temperatura, vento, sol, chuva, oxigênio dentre outros. Essa exposição degrada o solo tornando-o pobre nas características físicas, químicas e biológicas. Por sua vez, a persistência de cultivos com baixo revolvimento do solo pode melhorar as propriedades do solo. Há grande relação entre maior disponibilidade de nutrientes a longo prazo no solo quando o mesmo persiste em sistema conservacionista. O aumento dos teores de matéria orgânica no solo mantém maior capacidade de troca de cátions o que garante melhor aporte de nutrientes para o solo, devido estarem intimamente ligados (SANTIAGO et al., 2008).

A máxima eficiência na correção da acidez do solo e na disponibilização de nutrientes essenciais (como o Ca e $\mathrm{Mg}$ ) pode ser garantida quando da aplicação de corretivo de solo e sua eficiência na homogeneização. Por outro lado, a prática de revolver o solo pode causar perdas indesejáveis como a perda de carbono orgânico e de água no solo, resultado do aumento da mineralização da matéria orgânica do solo e da desagregação do solo. Desse modo, visando promover o adequado preparo do solo para o cultivo da cana-de-açúcar de forma sustentável, econômica, eficiente e responsivo para a cultura desenvolveu-se algumas opções de preparo envolvendo grades aradora e niveladora, arado, subsolador e plantio direto.

Diante do exposto, o objetivo deste estudo foi avaliar o efeito de diversos tipos de preparo de solo e do plantio direto nas características agronômicas e produtividade da cana-de-açúcar em área de reforma na região do cerrado.

\section{Material e Métodos}

O trabalho foi realizado na usina Jalles Machado em Goianésia - GO $\left(15^{\circ} 10^{\prime}\right.$ de latitude sul e $49^{\circ} 15^{\prime}$ de longitude oeste, com aproximadamente $640 \mathrm{~m}$ de altitude). Antes do início do experimento, a área foi cultivada com as culturas de arroz, soja e milho e nos seis últimos anos foi cultivada com a cana-de-açúcar (Saccharum spp.), caracterizando área de reforma do canavial.

O clima da região, segundo a classificação de Köppen, é do tipo Aw (tropical de savana), com invernos secos e verões chuvosos e temperaturas médias variando de $23,7^{\circ} \mathrm{C}$ a $25,4^{\circ} \mathrm{C}$. Durante a condução do experimento ocorreram variações de temperatura de $21,8{ }^{\circ} \mathrm{C}$, no mês de junho, a $26,1^{\circ} \mathrm{C}$ em setembro e 
dezembro de 2009. A precipitação pluvial média anual é de $1500 \mathrm{~mm}$. Segundo aferições realizadas na usina ocorreram precipitações de $1435 \mathrm{~mm}$ durante o ano de 2009 e $570 \mathrm{~mm}$ nos primeiros meses do ano de 2010.

A implantação do experimento ocorreu em janeiro de 2009, realizando-se a dessecação da área, os manejos de preparo do solo os quais caracterizaram os tratamentos avaliados, se estendendo até julho de 2009. Amostras de solo classificado como Latossolo Vermelho-Amarelo (EMBRAPA, 2006) foram retiradas em duas profundidades e levadas para análises no laboratório da usina Jalles Machado antes da implantação do experimento. A análise química na profundidade de 0,0 a $0,20 \mathrm{~m}$ apresentou os seguintes resultados: $\mathrm{pH}$ em água de 5,$15 ; 1,30 \mathrm{mg} \mathrm{dm}^{-3}$ de $\mathrm{P} ; 54$ $\mathrm{mg} \mathrm{dm}{ }^{-3}$ de $\mathrm{K} ; 1,73 \mathrm{cmol}_{\mathrm{c}} \mathrm{dm}^{-3}$ de Ca; 0,66 $\mathrm{cmol}_{\mathrm{c}} \mathrm{dm}^{-3}$ de $\mathrm{Mg} ; 0,02 \mathrm{cmol}_{\mathrm{c}} \mathrm{dm}^{-3}$ de $\mathrm{Al} ; 2,54 \mathrm{cmol}_{\mathrm{c}} \mathrm{dm}^{-3}$ de $\mathrm{H}+\mathrm{Al}$; saturação de $\mathrm{Al} 1,38 \%$; saturação de bases de 49,7\%; $1,93 \mathrm{~g} \mathrm{~kg}^{-1}$ de matéria orgânica e $431 \mathrm{~g} \mathrm{~kg}^{-1} \mathrm{de}$ argila. Na camada de 0,20 a 0,40 m apresentou: $\mathrm{pH}$ em água de 4,63;0,95 $\mathrm{mg} \mathrm{dm}^{-3}$ de $\mathrm{P} ; 6,8 \mathrm{mg} \mathrm{dm}^{-3}$ de $\mathrm{K}$; 0,45 $\mathrm{cmol}_{\mathrm{c}} \mathrm{dm}^{-3}$ de $\mathrm{Ca} ; 0,32 \mathrm{cmol}_{\mathrm{c}} \mathrm{dm}^{-3}$ de $\mathrm{Mg} ; 0,37$ $\mathrm{cmol}_{\mathrm{c}} \mathrm{dm}^{-3}$ de $\mathrm{Al} ; 3,02 \mathrm{cmol}_{\mathrm{c}} \mathrm{dm}^{-3}$ de $\mathrm{H}+\mathrm{Al}$; saturação de Al 33\%; saturação de bases de 20,1\%; 1,39 $\mathrm{g} \mathrm{kg}^{-1}$ de matéria orgânica e $450 \mathrm{~g} \mathrm{~kg}^{-1}$ de argila.

$\mathrm{O}$ ensaio foi implantado em delineamento de blocos casualizados com seis tratamentos e quatro repetições. A área experimental constou de $34.505 \mathrm{~m}^{2}$, incluindo os carreadores. Cada repetição consistiu de seis parcelas, contendo cada uma $50 \mathrm{~m}$ de comprimento e $19,5 \mathrm{~m}$ de largura, sendo composta por 13 linhas de cana-deaçúcar espaçadas de 1,5 m. Separando os blocos e as parcelas, foram feitos carreadores com largura de 5,0 m, cujo propósito foi efetuar manobras com máquinas e implementos. Os tratamentos são apresentados na Tabela 1.

Neste estudo foram utilizados herbicidas de largo espectro, glyphosate juntamente com 2, 4-D, nas doses de 3,0 e 2,0 $\mathrm{L} \mathrm{ha}^{-1}$, respectivamente, 30 dias antes da implantação do experimento (Tabela 1). Foram aplicados 1,5 Mg ha-1 de calcário dolomítico (PRNT de $85 \%$ ), em todos os tratamentos. Na aração foi utilizado arado de aiveca, atingindo uma profundidade efetiva de 0,30 a $0,40 \mathrm{~m}$. Para a gradagem utilizou-se uma grade leve, atingindo a profundidade de 0,15 a $0,20 \mathrm{~m}$. A subsolagem foi realizada por meio de subsolador, atingindo a profundidade média de $0,40 \mathrm{~m}$. No plantio direto foi efetuado uma sulcação utilizando-se profundidades de 0,30 a $0,40 \mathrm{~m}$. A destruição das soqueiras feito pelo destruidor de soqueiras modelo "eliminador mecânico de soqueiras" o qual possui dois discos de corte 23", dois levantadores tipo relha em "V" com grelhas de extensão com sistema de destruição das soqueiras tipo rolo faca batedor com 20 facas. Realizouse uma gessagem após a implantação de todos os tratamentos. Nesta, a dose aplicada foi de $800 \mathrm{~kg} \mathrm{ha}^{-1}$ sendo distribuída a lanço. A adubação de plantio foi realizada no sulco com distribuição de $250 \mathrm{~kg} \mathrm{ha}^{-1}$ de fosfato monoamônico (MAP). Essa dose equivale a 120 $\mathrm{kg} \mathrm{ha}^{-1}$ de $\mathrm{P}_{2} \mathrm{O}_{5}$ e $27 \mathrm{~kg} \mathrm{ha}^{-1}$ de $\mathrm{N}$.

Foi utilizada a variedade IAC 87-3396, sendo o plantio realizado manualmente no dia 24 de abril de 2009, colocando-se de 15 a 20 gemas por metro numa profundidade de 0,30 a 0,40 m. Após a distribuição dos toletes de cana nos sulcos, realizou-se a cobertura dos mesmos. No dia 05/12/2009 foi realizada uma adubação de cobertura aplicando-se o fertilizante líquido 05-00-13 $+0,3 \%$ de $\mathrm{Zn}+0,3 \%$ de $\mathrm{B}$ na dose de $1000 \mathrm{~L} \mathrm{ha}^{-1}$.

Tabela 1. Manejos de preparo de solo utilizados na implantação do experimento de cana-de-açúcar no ano agrícola de 2009, em Goianésia - Goiás.

\begin{tabular}{cl}
\hline Tratamentos & Operações nos sistemas de preparo de solo \\
\hline $\mathrm{T} 1$ & $\begin{array}{l}\text { Dessecação (Glyphosate e } 2,4-\mathrm{D} \text {, nas doses de } 3,0 \text { e } 2,0 \mathrm{~L} \mathrm{ha}{ }^{-1} \text {, respectivamente); } 1,5 \mathrm{Mg} \mathrm{ha}^{-1} \mathrm{de}_{\text {calcário; }} \\
\text { arado de aiveca (atuando na profundidade média de } 0,35 \mathrm{~m} \text { a } 0,4 \mathrm{~m} \text { ) e grade leve (atuando na profundidade } \\
\text { média de } 0,15 \mathrm{~m} \text { a } 0,2 \mathrm{~m}) .\end{array}$ \\
$\mathrm{T} 2$ & $\begin{array}{l}3,5 \mathrm{t} \mathrm{ha}^{-1} \text { de calcário; subsolador (atuando na profundidade média de } 0,35 \mathrm{~m} \mathrm{a} 0,4 \mathrm{~m} \text { ) e grade leve (atuando } \\
\text { na profundidade média de } 0,15 \mathrm{~m} \text { a } 0,2 \mathrm{~m}) .\end{array}$
\end{tabular}

PD - Dessecação (Glyphosate e 2,4-D, nas doses de 3,0 e 2,0 $\mathrm{L} \mathrm{ha}^{-1}$, respectivamente); 1,5 $\mathrm{Mg} \mathrm{ha}^{-1} \mathrm{de}$ calcário; e sulcação direta realizada a partir da abertura dos sulcos de plantio com sulcador, atuando na profundidade média de $0,3 \mathrm{~m}$ a $0,4 \mathrm{~m}$.

Dessecação (Glyphosate e 2,4-D, nas doses de 3,0 e 2,0 L ha-1, respectivamente); 1,5 $\mathrm{Mg} \mathrm{ha}^{-1}$ de calcário; subsolador (atuando na profundidade média de $0,35 \mathrm{~m}$ a $0,4 \mathrm{~m}$ ) e sulcação direta realizada a partir da abertura dos sulcos de plantio com sulcador, atuando na profundidade média de $0,3 \mathrm{~m}$ a $0,4 \mathrm{~m}$.

T5 Destruidor de soqueira; $1,5 \mathrm{Mg} \mathrm{ha}^{-1}$ de calcário; subsolador (atuando na profundidade média de $0,35 \mathrm{~m} \mathrm{a}$ $0,40 \mathrm{~m})$

Destruidor de soqueira; $1,5 \mathrm{Mg} \mathrm{ha}^{-1}$ de calcário; grade leve (atuando na profundidade média de $0,15 \mathrm{~m} \mathrm{a}$ $0,20 \mathrm{~m}$ ); arado de aiveca (atuando na profundidade média de $0,35 \mathrm{~m}$ a $0,40 \mathrm{~m}$ ) e grade leve (atuando na profundidade média de $0,15 \mathrm{~m}$ a $0,20 \mathrm{~m}$ ). 
Amostras foliares foram coletadas quando o canavial encontrava-se com nove meses após plantio. Cada amostra composta foi formada por 20 folhas, sendo cada folha originada de uma planta que foi escolhida aleatoriamente nas cinco linhas centrais da parcela, adotando-se a folha +1 , ou seja, a primeira com bainha visível a ser coletada.

A avaliação de número de colmos foi realizada dez meses após o plantio, contando-se todos os colmos compreendidos em $30 \mathrm{~m}$ das cinco linhas centrais, totalizando uma área avaliada de $225 \mathrm{~m}^{2}$.

As características agronômicas avaliadas foram diâmetro e altura do colmo. Foram selecionados dez colmos por parcela, na semana da colheita, escolhidos em sequência em uma linha central da parcela. O diâmetro $(\mathrm{cm})$ foi avaliado no terceiro entrenó da base para a ponta com paquímetro digital. A altura foi medida com fita métrica $(\mathrm{m})$ da base do colmo até o nó com a aurícula aberta.

Para a determinação da tonelada de colmos por hectare (TCH), o canavial foi queimado e o corte realizado manualmente. Posteriormente, determinou-se a massa de colmos das cinco linhas centrais de todos os $50 \mathrm{~m}$ da parcela, totalizando uma área avaliada de 375 $\mathrm{m}^{2}$ por parcela, sendo posteriormente os dados extrapolados para um hectare. A pesagem foi realizada com o auxílio de balança (Dinamômetro de pesagem marca Técnica modelo D-10000) adaptada a um carregador de cana.

Os dados foram submetidos a análise de variância pelo teste $\mathrm{F}$ a $5 \%$ de probabilidade, e as médias comparadas pelo teste de Tukey a 0,05 de nível de significância.

\section{Resultados e Discussão}

Com relação às características agronômicas da canade-açúcar, não foram encontradas diferenças entre os tratamentos (Tabela 2). Estes resultados indicam que a ausência de preparo de solo, como no tratamento plantio direto (PD), não influencia no desenvolvimento da canade-açúcar, principalmente nas fases da brotação, perfilhamento e crescimento. Apesar da diferença entre os preparos de solo, em todos os tratamentos, houve a prática da sulcação, que nada mais é do que abrir um sulco para a deposição do tolete. Portanto, em todas os tratamentos, a brotação e o perfilhamento da cana encontrou condições semelhantes. Camilotti et al. (2005) não encontraram diferenças no perfilhamento entre tratamentos que revolveram o solo em seu preparo e da mesma forma não constataram diferenças para o tratamento cultivo mínimo.

Tavares et al (2010) avaliando quatro preparos de solo, também não identificaram diferença para as características agronômicas da cana-de-açúcar. Camilotti et al. (2006) avaliando o efeito prolongado de preparo de solo em cana-de-açúcar não encontraram diferença para o número de perfilho entre os diferentes preparos. Segundo estes autores a brotação e o perfilhamento estão mais associados à característica das variedades do que ao sistema de preparo de solo. Barbieri et al. (1997) avaliaram diferentes preparos de solo e identificaram, inicialmente, maior brotação na área em que houve preparo convencional. Entretanto, no decorrer do ciclo, os tratamentos apresentaram números de colmos por área semelhantes.

Apesar de não haver diferenças para o número de colmos entre os tratamentos, houve diferença entre produtividade (Figura 1). Os tratamentos em que o revolvimento do solo foi mais intenso, com o uso de grades e do arado de aiveca, como na dessecação + calcário + aração + grade e na destruição de soqueira + calcário + grade + aração + grade foram os que obtiveram as maiores produtividades 104,9 e $105,3 \mathrm{Mg}$ $\mathrm{ha}^{-1}$, respectivamente. Houve diferença de $12 \mathrm{Mg} \mathrm{ha}^{-1}$ quando comparado às áreas em que o preparo do solo foi realizado com o subsolador sendo 93,5 e 93,7 Mg ha${ }^{1}$ para destruidor de soqueira + calcário + subsolador e dessecação + calcário + subsolador + grade, respectivamente. Entretanto, deve-se ressaltar que a dessecação + calcário (T3) não diferiu em produtividade de colmos em comparação aos tratamentos que receberam revolvimento mais intenso do solo. Este fato demonstra que, para as condições do ensaio, em canaplanta, é viável a realização do plantio direto da canade-açúcar em área de reforma de canavial.

Tabela 2. Características agronômicas da cana-de-açúcar submetida a diferentes preparos de solo, em área de renovação de canavial na região do cerrado, em maio de 2010 .

\begin{tabular}{lccc}
\hline Preparos de solo & $\begin{array}{c}\text { Diâmetro do colmo } \\
(\mathbf{c m})\end{array}$ & $\begin{array}{c}\text { Altura do colmo } \\
(\mathbf{m})\end{array}$ & $\begin{array}{c}\text { Número de colmos } \\
\text { por metro }\end{array}$ \\
\hline Dessecação + calcário + aração + grade & $2,68 \mathrm{a}$ & $2,54 \mathrm{a}$ & $14,87 \mathrm{a}$ \\
Dessecação + calcário + subsolador + grade & $2,58 \mathrm{a}$ & $2,37 \mathrm{a}$ & $14,87 \mathrm{a}$ \\
Dessecação + calcário (PD) & $2,61 \mathrm{a}$ & $2,43 \mathrm{a}$ & $15,37 \mathrm{a}$ \\
Dessecação + calcário + subsolador & $2,62 \mathrm{a}$ & $2,52 \mathrm{a}$ & $14,25 \mathrm{a}$ \\
Destruidor de soqueira + calcário + subsolador & $2,67 \mathrm{a}$ & $2,32 \mathrm{a}$ & $15,17 \mathrm{a}$ \\
Dest. de soqueira + calcário + grade + aração + grade & $2,62 \mathrm{a}$ & $2,42 \mathrm{a}$ & $14,95 \mathrm{a}$ \\
\hline CV $(\%)$ & 6,92 & 10,61 & 4,06 \\
\hline
\end{tabular}

Médias seguidas por letras diferentes diferem estatisticamente pelo teste $\mathrm{F}$ e Tukey a $5 \%$ de probabilidade. 
Normalmente, em trabalhos com preparos de solo na cultura da cana-de-açúcar não são detectadas diferenças entre os tratamentos para produtividade, como por exemplo em Tavares et al (2010) e Paulino et al. (2004). Este fato pode ocorrer pelo pequeno tamanho da área amostrada para determinação da produtividade e que gera um grande coeficiente de variação o qual não permite a detecção da diferença entre os tratamentos. Paulino et al. (2004) avaliando três formas de escarificação na entrelinha da soqueira, em uma área de $39,0 \mathrm{~m}^{2}$, cortaram dez colmos, pesaram, e com o peso médio do colmo, multiplicaram pelo o número de colmos da área. Estes autores obtiveram um valor de coeficiente de variação de $27 \%$ e diferença de $20 \mathrm{Mg}$ ha 1 .

Neste trabalho toda a cana cortada na área avaliada de $375 \mathrm{~m}^{2}$ foi pesada, podendo ser este o motivo do baixo $\mathrm{CV}$ e das diferenças encontradas. Grange et al. (2005) avaliando cinco preparos de solo, na Tailândia, em área de expansão e reforma de canavial e avaliando uma área de $80 \mathrm{~m}^{2}$, durante oito safras (cana-planta expansão + três socas - cana-planta - reforma + duas socas e cana-planta - reforma), encontraram mais do que o dobro da produtividade da área de plantio direto no primeiro ano de cana-planta para o tratamento convencional. As áreas, onde o preparo foi realizado com o subsolador obtiveram produtividade intermediária. Já nas reformas do canavial, o preparo que obteve maiores produtividades foi o que utilizou o subsolador. Neste trabalho, o PD apresentou-se pior nos anos em que os preparos de solo eram realizados. Porém, nas soqueiras, espera-se uma tendência de se equiparar aos demais tipos de preparos.

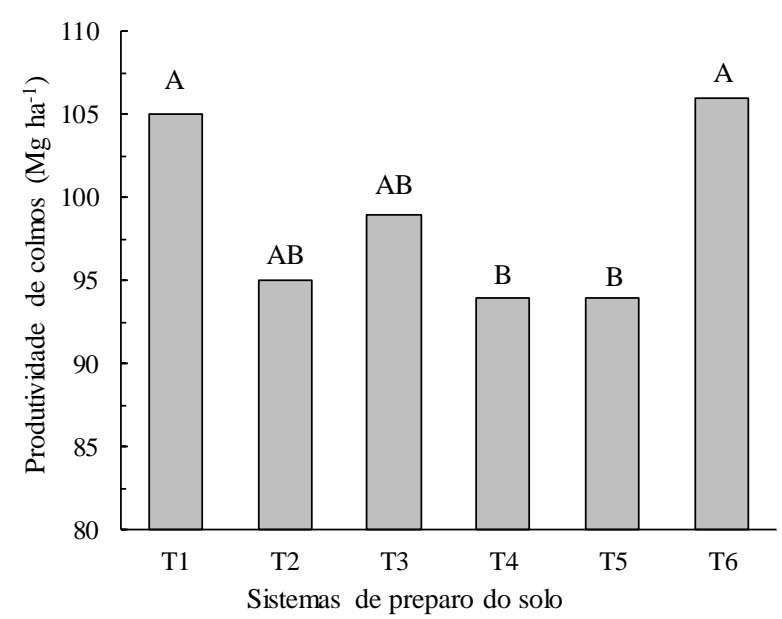

Figura 1. Produtividade de colmos de cana-de-açúcar em área de reforma submetida a diferentes formas de preparo de solo, no cerrado. Barras seguidas por letras diferentes diferem entre si pelo teste Tukey a $5 \%$ de probabilidade.

Legenda: T1 - Dessecação + calcário + aração + grade; T2 - Calcário + subsolador + grade; T3 - Dessecação + calcário + plantio direto; T4 - Dessecação + calcário + subsolador; T5 - Destruidor de soqueira + calcário + subsolador; e T6 - Destruidor de soqueira + calcário + grade + aração+ grade.
Camilotti et al. (2005) trabalharam com quatro manejos de preparo de solo onde um deles denominou de cultivo mínimo que foi apenas abertura do sulco e plantio das mudas e os demais tratamentos envolvendo o revolvimento do solo para posterior abertura do sulco e plantio. Não detectaram diferenças de produtividade em cana-planta.

Portanto, observa-se que para a renovação de canavial o uso de subsolador, precedido por destruidor de soqueiras ou não, é a opção menos vantajosa, pois obteve a menor produtividade da cana-planta. Já o PD aqui chamado, mobilizou apenas no sulco de plantio e apresentou-se como uma opção viável, pois, além de não diferir em produtividade, pode apresentar menores custos. Carvalho et al. (2011) encontraram R $\$ 83,00$ por ha de custos para o plantio da cana em sistema de plantio direto, ao contrário contra $\mathrm{R} \$ 445,00$ por ha no preparo do solo com uma gradagem pesada a $0,20 \mathrm{~m}$, adicionalmente a uma gradagem intermediária, seguida de uma aração e gradagem niveladora.

\section{Conclusões}

Os diferentes sistemas de preparo de solo não influenciam nas características agronômicas da canaplanta.

$\mathrm{O}$ uso de arado de aiveca associado à grade na incorporação do calcário proporciona as maiores produtividades de colmos de cana-de-açúcar.

O plantio direto mostrou-se uma alternativa viável ao cultivo da cana-de-açúcar, por apresentar produtividade de colmos semelhante aos demais preparos do solo.

\section{Agradecimentos}

A FAPEMIG e à CAPES pelo apoio financeiro e a Usina Jalles Machado com sua equipe de pesquisa.

\section{Referências Bibliográficas}

ARRUDA, E. M.; ALMEIDA, R. F. DE.; SILVA-JUNIOR, A. C.; RIBEIRO, B. T.; SILVA, A. A.; LANA, R. M. Q. Aggregation and organic matter in different tillage systems for sugarcane. African Journal of Agricultural Research, Victoria Island Lagos, v.10, n. 4, p.281-288, 2015.

BARBIERI, J. L.; ALLEONI, L. R.; DONZELLI, J. L. Avaliação agronômica e econômica de sistemas de preparo de solo para cana-de-açúcar. Revista Brasileira de Ciência do Solo, Campinas-SP, v. 21, n.1, p. 89-98, 1997.

CAMILOTTI, F.; ANDRIOLI, T.; MARQUES, M. O.; SILVA, A. R.; TASSO JÚNIOR, L. C.; NOBILE, F. O. Atributos físicos de um Latossolo cultivado com cana-deaçúcar após aplicações de lodo de esgoto e vinhaça. Engenharia Agrícola. Jaboticabal-SP, v. 26, n. 3, p. 738-747. 2006. 
CAMILOTTI, F.; ANDRIOLI, I.; DIAS, F. L. F.; CASAGRANDE, A. A., SILVA, A. R.; MUTTON, M. A.; CENTURION, J. F. Efeito prolongado de sistemas de preparo do solo com e sem cultivo de soqueira de cana crua em algumas propriedades físicas do solo. Engenharia Agrícola, Jaboticabal-SP, v.25, n.1, p. 189-198, 2005.

CARVALHO, L.A.; SILVA-JUNIOR, C.A.; NUNES, W. A. G. A.; MEURER, I.; SOUZA-JÚNIOR, W. S. Produtividade e viabilidade econômica da cana-de-açúcar em diferentes sistemas de preparo do solo no centro-oeste do Brasil. Revista de Ciências Agrárias, Lisboa, v. 34, n.1, p. 199-211, 2011.

CHAVES, V. A.; SANTOS, S. G.; SCHULTZ, N.; PEREIRA, W.; SOUSA, J. S.; MONTEIRO, R. C.; REIS, V. M. Desenvolvimento inicial de duas variedades de cana-de-açúcar inoculadas com bactérias diazotróficas. Revista Brasileira Ciência do Solo, Viçosa-MG, v. 39, n. 6, p. 1595-1602, 2015.

CONAB. COMPANHIA NACIONAL DE ABASTECIMENTO. Terceiro levantamento da safra brasileira - Cana-de-açúcar. Brasília: Conab, 2015. Disponível em: < http://www.conab.gov.br/ >. Acesso em: 05 de jan. 2016.

EMBRAPA. EMPRESA BRASILEIRA DE PESQUISA AGROPECUÁRIA. Sistema Brasileiro de classificação de Solos. 2 ed. Embrapa Solos. Rio de Janeiro, RJ. 306 p. 2006.
INÁCIO, J. B.; SANTOS, R. J. A expansão canavieira no município de delta- MG: "ilhados pelos canaviais". Caminhos de Geografia, Uberlândia-MG, v. 14, n. 48, p. 209-227, 2013.

GRANGE, I.; PRAMMANEE, P.; PRASERTSAK, P. Comparative analysis of different tillage systems used in sugarcane (Thailand). Australian Farm Business Management Journal, Melbourne, v. 2, n. 1, p. 46-50, 2005.

NEVES, M. F.; CONEJERO, M. A. Sistema agroindustrial da cana: cenários e agenda estratégica. Economia Aplicada, São Paulo-SP, v. 11, n. 4, p. 587-604, 2007.

PAULINO, A. F.; MEDINA, C. C.; AZEVEDO, M. C. B.; SILVEIRA, K. R. P.; TREVISAN, A. A.; MURATA, I. M. Escarificação de um latossolo vermelho na pós-colheita de soqueira de cana-de-açúcar. Revista Brasileira de Ciência do Solo, Viçosa-MG, v. 28. p. 911-917, 2004.

SANTIAGO, A.; QUITERO, J. M.; DELGADO, A. Longterm effects of tillage on the availability of iron, copper, manganese, and zinc in a Spanish Vertisol. Soil \& Tillage Research, Seville, v. 98, n 2, p. 200-207, 2008.

TAVARES, O. C. H.; LIMA, E.; ZONTA, E. Crescimento e produtividade da cana planta cultivada em diferentes sistemas de preparo do solo e de colheita. Acta Scientiarum Agronomy, Maringá-PR, v. 32, n. 1, p.61-68, 2010. 